\title{
ESPACIO MULTISENSORIAL PARA LA ESTIMULACIÓN DE NIÑOS ENTRE DOS Y CINCO AÑOS CON DÉFICIT SENSORIAL
}

\author{
Rommel Fernando Morocho Ortega ${ }^{1}$, Kenneth Enrique Tacuri \\ Mora $^{2}$, Juan Pablo Pazmiño ${ }^{3}$, Daniel Claudio Perazzo Logioia ${ }^{+}$ \\ ${ }^{1}$ Tecnólogo en Desarrollo de Software egresado del Instituto Superior \\ Tecnológico Particular Sudamericano, Cuenca \\ E-mail: rommelmorocho@hotmail.es (ID) https://orcid.org/0000-0001-7486-8062 \\ ${ }^{2}$ Tecnólogo en Desarrollo de Software egresado del Instituto Superior \\ Tecnológico Particular Sudamericano, Cuenca \\ E-mail: ktmnobleh@gmail.com (D) https://orcid.org/0000-0003-0630-5035 \\ ${ }^{3}$ Tecnólogo en Desarrollo de Software egresado del Instituto Superior \\ Tecnológico Particular Sudamericano, Cuenca \\ E-mail: jppp74@hotmail.com (D) https://orcid.org/0000-0003-0069-7680 \\ + Vicerrector del Instituto Superior Tecnológico Particular Sudamericano, Cuenca \\ E-mail: vicerectoradoits@sudamericano.edu.ec \\ (D) https://orcid.org/0000-0003-3089-2225
}

\section{RESUMEN}

La estimulación sensorial busca enriquecer la experiencia sensorial del niño exponiéndole a una serie de estímulos controlados. Las primeras aulas multisensoriales datan de los años 70, y hoy en día se continúan diseñando. En la actualidad la manera de abordar la atención educativa a niños con necesidades especiales se está enriqueciendo al incorporarse numerosas investigaciones que avalan la eficacia del uso de alternativas digitales que mejoran las prestaciones de las aulas y proporcionan nuevas experiencias a los usuarios. Este artículo expone los resultados de la investigación desarrolla de manera conjunta entre estudiantes y docentes del Instituto Superior Tecnológico Particular Sudamericano como parte de un proyecto de investigación grupal que permite la incorporación del trabajo de titulación presentado para optar al título de Tecnólogos en Desarrollo de Software. La investigación tuvo como objetivo el diseño de un espacio multisensorial basado en estrategias cognitivas para la estimulación de niños entre dos y cinco años con déficit sensorial para facilitar el desarrollo de tareas cotidianas sencillas y el inicio del desenvolvimiento dentro de su entorno. El diseño se logró mediante la asesoría de expertos en las áreas de psicología, psicopedagogía, entre otras. En cuanto a la estructura física del espacio, este está subdividido en rincones (módulos) con el propósito de conformar un espacio multisensorial que permite estimular los sentidos del niño, atendiendo a su deficiencia psicomotriz. El proyecto se llevó a cabo con una metodología teórico-práctica, para lo cual se utilizó información documental de diversas investigaciones en las que afirman que la estimulación sensorial es esencial para el desenvolvimiento de los niños en sus primeras etapas de vida. Toda esta información recolectada orientó para el desarrollo de los módulos multisensoriales para hacerlos funcionales. Como resultado se obtiene la cons- 
trucción y propuesta de aplicabilidad del espacio multisensorial en instituciones que atiendan niños de 2 a 5 años con déficit de atención. Los módulos creados están destinados a convertir- se en una herramienta educativa, para niños en etapas iniciales de la vida.

Palabras Clave: espacio multisensorial, estimulación, niños, déficit sensorial

\section{ABSTRACT}

Sensory stimulation seeks to enrich the child's sensory experience by exposing him to a series of controlled stimuli. The first multisensory classrooms date back to the 1970s, and are still being designed today. At present, the way of approaching educational care for children with special needs is being enriched by incorporating numerous investigations that support the effectiveness of the use of digital alternatives that improve the performance of the classroom and provide new experiences to users. This article presents the results of the research developed jointly by students and teachers of the Instituto Superior Tecnológico Particular Sudamericano as part of a group research project that allows the incorporation of the degree work presented to qualify for the title of Technologists in Software Development. The research aimed at designing a space through cognitive strategies for the stimulation of children between two and five years old with sensory deficits to facilitate the development of simple daily tasks and the beginning of development within their environment. The design was achieved through the advice of experts in the areas of psychology, psychopedagogy, among others. As for the physical structure of the space, it is subdivided into corners (modules) with the purpose of forming a multisensory space that allows the child's senses to be stimulated, attending to his psychomotor deficiency. The project was carried out with a theoretical-practical methodology, for which documentary information from various investigations was used in which they affirm that sensory stimulation is essential for the development of children in their early stages of life. All this information collected guided the development of multisensory modules to make them functional. As a result, the construction and proposal of applicability of the multisensory space is obtained in institutions that serve children from 2 to 5 years with attention deficit. The modules created are intended to become an educational tool for children in the initial stages of life.

Keywords: multisensory space, stimulation, children, sensory deficit

Forma sugerida de citar: Morocho Ortega, R. F., Tacuri Mora, K. E., Pazmiño, J. P. \& Perazzo Logioia, D. C. (2021). Espacio multisensorial para la estimulación de niños entre dos y cinco años con déficit sensorial. Convergence Tech Revista Científica. 5(1), 61-76. https://doi.org/10.53592/convtech.v5iV.2

\section{INTRODUCCIÓN}

La estimulación temprana favorece al desarrollo neuronal del individuo, ya que permite el mayor desarrollo de las conexiones cerebrales (Aguilar et al., 2012). Esta estrategia se fundamenta en la repetición a partir del estímulo de los sentidos; de esta manera, se refuerzan áreas neuronales de interés. Por ejemplo, el refuerzo del área motora es a través del gateo, en el cual un bebé debe ser capaz de coordinar la mano derecha con el pie izquierdo (patrón cruzado) (Vega, 2016).
De acuerdo con el diagnóstico realizado para la investigación, en la ciudad de Cuenca existen centros para la estimulación temprana de niños con capacidades especiales. En la mayoría de estos Centros utilizan métodos tradicionales para la estimulación debido a que desconocen o no cuentan con los recursos necesarios para adquirir nuevas tecnologías que presenta mejores resultados, por lo cual, es importante proponer módulos o espacios multisensoriales que promuevan el reemplazo de estas prácticas para agilizar los procesos actuales. 
Dentro de los proyectos del Instituto Particular Tecnológico Sudamericano existe la línea de investigación enfocada hacia la automatización y control, sumado esto al dominio académico en educación nace el proyecto de investigación institucional al cual se integran los estudiantes de la carrera de Desarrollo de Software para el desarrollo de los módulos descritos en el siguiente documento.

Los sentidos nos permiten percibir, conocer y relacionarnos con el medio que nos rodea. Debido a que estos son los encargados de captar los estímulos y convertidos en señales para ser enviadas al sistema nervios en donde son procesadas, a su vez el sistema nervioso elabora respuestas a ser ejecutadas ante estos estímulos. Por lo cual, se propone el diseño de módulos multisensoriales que permitan estimular el desarrollo de los sentidos y capacidades motrices del niño. Además, el proyecto tiene como finalidad ofrecer una nueva herramienta para centros educativos, enfocada en los resultados obtenidos de cada interacción con los módulos y el sistema, permitiendo así a terapeutas tener un control más exacto del progreso de desarrollo intelectual y físico del niño.

En los centros de educación especial es esencial ofrecer un proceso de enseñanza basada en la Estimulación Multisensorial así se proporciona estímulos para los diferentes canales sensoriales y de esta forma el niño puede interpretarlos y asociarlos con el entorno. En la actualidad los estudios e investigaciones realizadas muestran excelentes resultados en la utilización de estímulos multisensoriales controlados.

Todo lo anterior sustenta la importancia del desarrollo de módulos multisensoriales para la estimulación, que son de gran ayuda en el aprendizaje y desarrollo de los niños con capacidades especiales porque de cierta forma les ayuda a entrenar su mente y coordinar sus movimientos a través de la percepción de diferentes estímulos visuales, auditivos, táctiles, entre otros.

El contar con un sistema de seguimiento proporcionara mejor control del avance y progreso del niño. En el sistema se puede llevar un historial de cada sesión y de esta forma evaluar si los métodos aplicados en las terapias están dando buenos resultados. Además, se le proporcionará una herramienta móvil que le permita controlar los módulos de manera que cuando el niño interactúe con el módulo se puede variar algunos parámetros como colores y sonidos. La aplicación de nuevas tecnologías en centros de educación especial serán un importante aporte para el aprendizaje y desarrollo de los niños.

\section{DESARROLLO}

El aula multisensorial o los módulos multisensoriales van más allá de ser una simple herramienta educativa para los alumnos con necesidades educativas especiales, es una terapia estimulativa controlada cuyo objetivo es la integración de los sentidos y la mejora de calidad de vida en todas aquellas personas con un déficit físico o psíquico específico (Gómez, 2012).

El desarrollo psicomotor de un niño tiene como fin alcanzar distintas habilidades que conforme pasen los años se vayan perfeccionando. En presencia de una alteración, este proceso se verá afectado, presentando repercusiones a corto o largo plazo en el desempeño del ser humano. En el caso de los niños que presentan discapacidad intelectual este desarrollo es más lento, provocando limitaciones físico-motoras, sensoriales o conductuales. Entre los aspectos que se encuentran alterados está el equilibrio, lo que repercute en la capacidad de desenvolverse de manera segura e independiente en cualquier entorno y bajo cualquier circunstancia. La estimulación multisensorial es uno de los métodos que se utiliza para trabajar con estos niños por sus buenos resultados a nivel cognitivo, conductual y emocional (Álvarez, 2016)

El termino estimulación sensorial hace referencia a toda la información del entorno que ingresa al sistema nervioso a través de los sentidos y a su vez esta información es procesada y transformada en percepciones y sensaciones (Katerinesisalima et al., 2013). De tal manera que, esta es una fase principal ya que a partir de ella se construye cualquier tipo de aprendizaje y permite el desarrollo de las funciones cognitivas como: la atención, la memoria, la capacidad de resolución de problemas, el razonamiento y la creatividad.

A continuación, se hace referencia a los diferentes tipos de estimulación y sus componentes más importantes.

La estimulación visual infantil: tiene por objetivo ayudar al niño a desarrollar todas sus capacidades físicas y emocionales. Los niños utilizan la capacidad visual desde que nacen para explorar progresivamente su 
entorno. El sentido de la vista les brinda información necesaria sobre el tamaño, la forma, los colores de los objetos, de las personas de su alrededor y de los movimientos que realizan. Los estímulos visuales que reciben les incentivan a conquistar el espacio o en otras palabras estos estímulos pueden generar reacciones como mover un objeto y a sonreír a su madre cuando se acerca (Coronel, 2014).

Una buena visión ayuda a mejorar su coordinación ojo-mano tomando los objetos a su alcance o buscándolos con la mirada. Dentro de las recomendaciones para el desarrollo y la estimulación visual de un niño se encuentran segmentados en meses-edades (Santos, 2012).

Recomendaciones para la estimulación de un niño de acuerdo a la edad (Hernández, 2019)

- 1 - 3 meses: Presente objetos de muchos colores, llamativos y móviles. Desplace objetos en sentido horizontal a la altura de sus ojos para que los siga con la mirada.

- 4-6 meses: Haga gestos que el niño pueda imitar. Muéstrele sus propias manos y pies para que los reconozca. Presente objetos familiares como juguetes preferidos.

- 7 - 9 meses: Esconda objetos y ayúdele a encontrarlos. Obsequie pelotas suaves para aventar. Permita que toque y observe objetos con diferentes formas y texturas.

- 10 - 12 meses: Estimule la percepción con profundidad usando una caja de cartón como túnel. Permítale gatear antes de comenzar a caminar para que refuerce la coordinación del ojo - mano - pie - cuerpo. Ponga al alcance de su hijo recipientes con objeto para introducir y sacar juegos para embonar formas geométricas sencillas.

- 2 - 3 años: Proporciónele materiales para garabatear con crayolas de colores. Cuentos con imágenes y texturas.
- 4 - 5 años: Estimúlelo a que realice dibujos complejos y de completar. Permítale explorar al aire libre. Proporciónele materiales para construir juguetes. Ponga al alcance del niño juegos que involucren la realización de laberintos, rompecabezas y memorias.

La estimulación de la audición: tiene muchos beneficios, por ejemplo, le ayuda a hablar antes. Al hablarle a un niño le ayuda a reconocer la voz de una persona y si se le presentan objetos reconocerá esos objetos. Hacer Distintas voces, reproducir tonos, canciones y hasta reír es un estímulo auditivo.

La voz de la madre le calma y le tranquiliza desde que nace, igual que el latido del corazón, diversos estudios han revelado que el bebé puede escuchar desde el útero materno. Al principio, son las voces humanas los sonidos que más atraen la atención del bebé y es importante saber utilizar este recurso para llamar su atención con distintas voces, tonos, canciones y risas.

- Recién nacidos. Ante un sonido brusco, seguramente se despertará. Si le gusta, agitará sus brazos y emitirá algún sonido como respuesta. El estímulo sonoro preferido en estos primeros momentos es la voz materna, que no sólo le estimulan, sino que también le tranquilizan.

- Cuatro meses. Buscará la procedencia del sonido con la mirada para ver qué lo produce. Al principio, la búsqueda es muy rudimentaria y después se mostrará cada vez más seguro sólo en el plano lateral.

- Cinco a seis meses de vida. Comienza a imitar con vocalizaciones los sonidos de los adultos.

- Segundo semestre. Busca lateralmente y hacia abajo el objeto o la persona que emitió el sonido. Alrededor de los 12 meses, hacia arriba.

- Año y medio. Es capaz de encontrar la fuente sonora en cualquier dirección: arriba, abajo y a sus costados.

- Veinticuatro meses. Localiza los sonidos en todos los ángulos. 


\section{Alteración o trastorno sensorial}

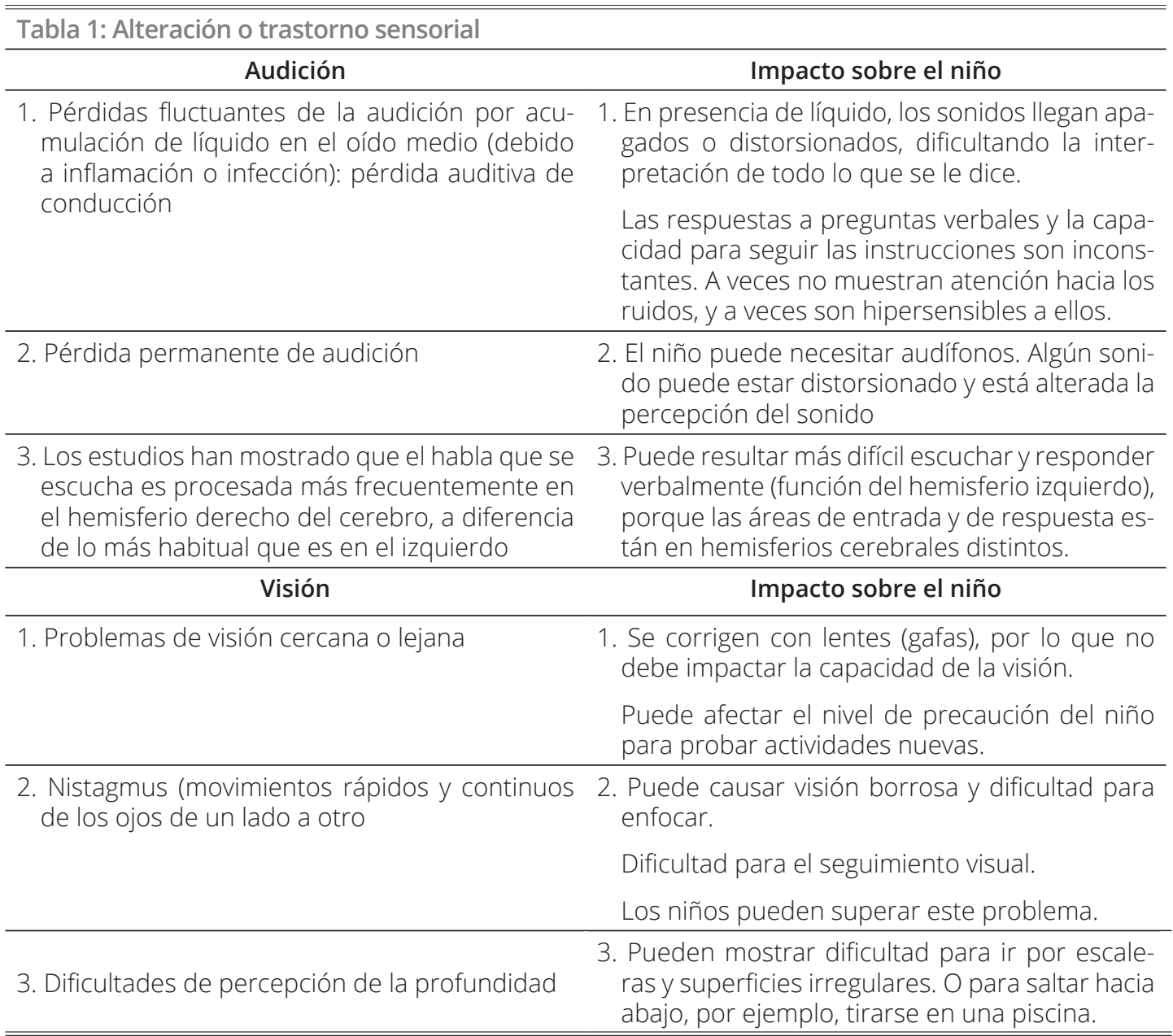

Estimulación Táctil: diferencia de la estimulación visual o auditiva la estimulación táctil no se aplica en todo el cuerpo si no que actúa directamente con las manos o la cara de un niño siendo las primeras en interactuar para descubrir un entorno. El estímulo táctil se da a partir de que el individuo entre en contacto con alguna superficie, la misma que puede contener forma y estar cubierta de texturas, esto es ayudará a diferenciar objetos, formas y texturas (Miranda \& Yracema, 2017).

Entre los beneficios de la estimulación táctil son conocer el mundo proporcionando información de los diferentes ambientes, seguridad, Conocer las diferencias entre blando y duro, para el momento de que empiecen a gatear y caminar (Miranda \& Yracema, 2017).

El estímulo del tacto proporciona protección ya que genera respuestas a estímulos potencialmente nocivos con movimiento, se aumenta el nivel de alerta, y emociones negativas. Como también de discriminación ya que permite la interpretación de las características temporales y espaciales del estímulo para funciones cognitivas (Miranda \& Yracema, 2017)..

\section{Psicomotricidad en el ámbito neuronal}

El desarrollo psicomotor constituye un área crucial dentro del desarrollo global del niño, entendido este como un proceso dinámico, sumamente complejo, fruto de la interacción entre factores genéticos y ambientales, que se sustenta en la evolución biológica, psicológica y social, dando como resultado la maduración orgánica y funcional del sistema nervioso, el desarrollo de funciones psíquicas y la estructuración de la personalidad. Así, el movimiento del niño favorece un óptimo autoconocimiento basado en la comunicación interpersonal, el dominio del 
lenguaje gestual y el autocontrol a través de los cuáles interpreta el mundo (ASIN, 1989)

La psicomotricidad se propone, como objetivo general, desarrollar o restablecer, mediante un abordaje corporal (a través del movimiento, la postura, la acción y el gesto), las capacidades del individuo. Podíamos incluso decir que pretende llegar por la vía corporal al desarro- llo de las diferentes aptitudes y potencialidades del sujeto en todos sus aspectos (motor, afectivo-social, comunicativo-lingüístico, intelectual-cognitivo) (BERRUEZO ADELANTADO, 2008).

La psicomotricidad busca activar los diferentes sentidos de una persona mediante la estimulación de los músculos.

\begin{tabular}{|c|c|c|}
\hline \multicolumn{3}{|c|}{ FASES DE LA ESTRUCTURACIÓN DEL ESQUEMA CORPORAL } \\
\hline $\begin{array}{l}\text { CUERPO VIVIDO } \\
\text { (0-3 años) }\end{array}$ & $\begin{array}{l}\text { Maduración de los cen- } \text { Ley céfalo-caudal. } \\
\text { tros motores (mieliniza- Ley próximo-distal } \\
\text { ción de fibras nerviosas). }\end{array}$ & $\begin{array}{l}\text { Control del propio cuer- } \\
\text { po (motricidad global). }\end{array}$ \\
\hline $\begin{array}{l}\text { CUERPO PERCIBIDO } \\
\text { (3-7 años) }\end{array}$ & $\begin{array}{l}\text { Percepción e } \text { interio- } \text { Motricidad transitiva. } \\
\text { rización del propio } \\
\text { cuerpo. Percepción del } \\
\text { mundo externo. }\end{array}$ & $\begin{array}{l}\text { Control del cuerpo (glo- } \\
\text { bal y segmentario) }\end{array}$ \\
\hline $\begin{array}{l}\text { CUERPO } \\
\text { REPRESENTADO } \\
\text { (7-12 años) }\end{array}$ & $\begin{array}{l}\text { Acción interiorizada y Acomodamiento práxico. } \\
\text { reversible. }\end{array}$ & $\begin{array}{l}\text { Conciencia del propio } \\
\text { cuerpo. }\end{array}$ \\
\hline
\end{tabular}

\section{Bases de la actividad Motriz}

Las bases fundamentales para la actividad motriz:

- Postura es la actividad que refleja el cuerpo con relación al espacio. Posición es la postura característica de una especie.

- La actitud guarda relación con los reflejos (de cierta intencionalidad) que producen la vuelta a una posición específica de la especie.

- Equilibrio es la interacción entre varias fuerzas, especialmente la de gravedad, y la fuerza motriz de los músculos esqueléticos.

Un organismo alcanza el equilibrio cuando puede mantener y controlar posturas, posiciones y actitudes. La postura se basa en el tono muscular y el equilibrio se basa en la propioceptividad (sensibilidad profunda), la función vestibular y la visión, siendo el cerebelo el principal coordinador de esta información. La postura se relaciona principalmente con el cuerpo, mientras que el equilibrio se relaciona principalmente con el espacio (Schrager, 1986). El equilibrio útil es la posición que permite los procesos de aprendizaje natural: aquellas habilidades necesarias para la supervivencia de la especie y la incorporación de gran cantidad de información externa.
Por tanto, postura y equilibrio son, a la vez que la base de las actividades motrices, la plataforma donde se apoyan los procesos de aprendizaje (Schrager, 1986).

La postura y el equilibrio son las bases fundamentales de la actividad psicomotriz ya que son las bases de futuras actividades cotidianas de una persona.

\section{Enseñanza Multisensorial}

La enseñanza multisensorial es una metodología alternativa que incluye a las diferentes inteligencias y estilos de aprendizaje en donde se involucra más de un sentido a la vez. Actualmente predomina el método de enseñanza en el que la vista y el oído son los principales y únicos receptores del aprendizaje, y no se ocupan otras experiencias de otro tipo. Con el método multisensorial se introducen varios sentidos en un mismo aprendizaje y con ello se multiplican las posibilidades y las oportunidades de un aprendizaje mejor y más completo.

Estudios realizados por el National Institutes of Child Health and Human Development (Instituto Nacional de Salud Infantil y Desarrollo Humano) han demostrado que este método de enseñanza multisensorial es el más eficaz para conseguir que los niños con dificultades en lecto-escritura las superen. 
En el aprendizaje de la lectura y la escritura se hacen firmes vínculos entre lo que vemos, lo que oímos, lo que palpamos. Los profesores que utilizan este enfoque o método enseñan a los niños a relacionar los sonidos de las letras con la escritura de la misma. Y a medida que los niños aprenden una nueva letra o patrón, cuidadosamente trazan, copian y escriben la letra al mismo tiempo que emiten su sonido correspondiente.

\section{La inteligencia y estimulación psicomotriz}

La movilidad humana inició cuando los seres humanos empezaron a caminar erguidos con las manos libres para poder usar herramientas, esto les ha ido haciendo cada vez más inteligentes. Así es como la raza humana tuvo la capacidad de crear herramientas y cada vez más complejas que le ayudaron a aprender y comprender más sobre la tierra. Esta evolución nos ha convertido en los seres vivos más inteligentes y brillantes que habitamos el planeta (GLENN DOMAN , DOUGLAS DOMAN, 2012).

Esta elevada inteligencia tiene sus principales orígenes en la movilidad y en la función manual humanas. Ambas han ganado con cada década de evolución en habilidad, belleza, complejidad y esplendor (GLENN DOMAN, DOUGLAS DOMAN, 2012).

La Movilidad está firmemente unida a la inteligencia, debido a que la movilidad es una de los seis tipos de Inteligencia Humana, está firme unión es muy importante en la etapa en la que somos bebes y niños pequeños. La movilidad desempeña un papel muy importante dentro de nuestras vidas.

\section{Tipos de Inteligencia Humana}

Inteligencia se define como la capacidad de entender o comprender, pero no hay una sola sino varios tipos, muchas veces depende de los valores o la cultura de cada sociedad, otras de las funciones, aptitudes o talentos (Gardner, 2016).

Tradicionalmente se ha considerado que la inteligencia es una sola en cada uno de nosotros y en diferentes grados. De acuerdo a las teorías acerca de la inteligencia humana del psicólogo estadounidense Howard Gardner plantea que la inteligencia es la capacidad desarrollable y no es solo la capacidad de resolver problemas o elaborar productos que sean valiosos en una cultura. Esta teoría formula el término de "inteligencias múltiples", en el cual se agrupan 8 tipos de ellas.

Para definir cada uno de los tipos de inteligencias (Gardner, 2016) estudió el desarrollo de las habilidades de los niños y lo que sucede en casos de daño cerebral para observar cómo se manifiesta cada una de las inteligencias.

\section{Funciones que caracterizan al ser Humano}

Existen exactamente seis funciones que caracterizan a los humanos y que los hacen diferentes de todas las demás criaturas. Estas seis funciones son únicas en los seres humanos y todas ellas se realizan exclusivamente en la corteza cerebral humana, o como se denomina en ocasiones, neo corteza. De todas las criaturas, los humanos y solo los humanos han sido dotados de esta corteza cerebral única (el nivel de desarrollo más alto del cerebro antiguo) y también solo ellos se benefician de estas seis funciones exclusivas de esa parte del cerebro. Tres de esas funciones son de naturaleza motora y dependen completamente de las otras tres, que son de naturaleza sensorial (GLENN DOMAN , DOUGLAS DOMAN, 2012).

\section{Funciones Motoras}

- Caminar, correr y saltar en posición erguida y con un verdadero patrón cruzado de extremidades opuestas que se mueven a la vez (brazo derecho-pierna izquierda y brazo izquierdo-pierna derecha).

- Hablar con un lenguaje artificial, inventado, simbólico y vocal

- Escribir con un lenguaje artificial, inventado, simbólico y visual.

\section{Funciones Sensoriales}

- Ver, de tal forma que se pueda leer un lenguaje artificial, inventado, simbólico y visual.

- Oír, de tal forma que se pueda entender un lenguaje artificial, inventado, simbólico y vocal.

- Sentir, de tal forma que podamos identificar un objeto solo por el tacto, sin la confirmación de la vista, el olor o el gusto.

Basándose en estas seis funciones los neurólogos pueden medir y establecer la normalidad. Al niño se le hacen pruebas en relación con: 
- Su competencia en movilidad (caminar).

- Su competencia lingüística (hablar).

- Su competencia en habilidades manuales (escribir).

- Su competencia visual (comprensión lectora y visual).

- Su competencia auditiva (comprensión auditiva).

- Su competencia táctil (compresión mediante el tacto).

Pero estas pruebas no pueden ser realizadas hasta que el niño cumpla los seis años y pueda adquirir estas capacidades ya que esta edad el crecimiento del cerebro se ha completado. El más sorprendente de nuestros descubrimientos fue el hecho de que la excelencia en estas seis funciones de inteligencia no era heredada como todo el mundo creía, sino que en todas ellas era adquirida. Por tanto, el descubrimiento más feliz de todos fue que, ya que la excelencia era un producto de las experiencias del bebé, cualquier niño cuyos padres quisieran que su hijo adquiriera un nivel alto de movilidad podía alcanzarla (GLENN DOMAN , DOUGLAS DOMAN, 2012).

\section{Importancia de la Estimulación Psicomotriz en la Infancia}

Con fuertes bases teóricas y evidencias empíricas de gran sofisticación, en la actualidad, parece haber resurgido el interés por el estudio y la estimulación del desarrollo psicomotor desde la perspectiva ecológica, en particular, las comparaciones transculturales de los logros motores (Keller et al., 2002).

El desarrollo psicomotor constituye un área elemental dentro del desarrollo del niño, entendido este como un proceso dinámico y sumamente complejo resultado de la interacción de factores genéticos y ambientales que dan como resultado la maduración orgánica y funcional del sistema nervioso, el desarrollo de funciones psíquicas y la estructuración de la personalidad. Así, el movimiento del niño favorece a un óptimo autoconocimiento basado en la comunicación interpersonal, el dominio del lenguaje gestual y el autocontrol a través de los cuáles interpreta el mundo (Keller et al., 2002).

Las relaciones durante los primeros meses, son fundamentalmente motrices, se expresan a través del diálogo corporal influido por el tono y la motilidad espontánea y reactiva (Sánchez, 1989).

\section{El desarrollo Psicomotor como base para el aprendizaje}

El desarrollo psicomotor se refiere a los cambios en la habilidad del niño para controlar sus movimientos corporales desde sus primeros movimientos rígidos, excesivos, sin coordinar y pasos espontáneos hasta el control de movimientos más complejos, rítmicos, suaves y eficaces de flexión, extensión, locomoción etc.

En este sentido, (GLENN DOMAN , DOUGLAS DOMAN, 2012) proponen siete etapas significativas de desarrollo en la vida de un niño, durante todo el desarrollo del cerebro, desde el nacimiento hasta los seis años, que es cuando todos los procesos neurológicos humanos están funcionando a su eficiencia total.

\section{Construcción de los módulos para la estimulación de niños entre dos $y$ cinco años con déficit sensorial}

Los módulos sensoriales construidos están compuestos de una parte electrónica y una parte informática. La parte electrónica tiene como fin contribuir en los aspectos de movilidad, concentración, atención, aprendizaje e interacción con el terapeuta, además con el desarrollo auditivo, visual y táctil del niño usuario de los módulos. La parte informática que facilitará las tareas de interacción con los niños y control de los módulos de a través de un software de control y seguimiento en el cual el terapeuta podrá llevar un registro de toda la información obtenida en las terapias para su posterior análisis y evaluación. También contará con una aplicación móvil que le permitirá interactuar con los módulos cambiando sonidos, colores y secuencias de luces de los módulos todo esto con el objetivo de hacer la terapia más atractiva para el niño.

Para el control de los módulos fueron usados microcontroladores ya que son circuitos integrados con la capacidad de ejecutar ordenes programadas en su memoria. Uno de los microcontroladores más conocidos es la tarjeta de Arduino que se ajustaba a los requerimientos del proyecto.

Arduino Mega 2560 R3: Arduino Mega es una tarjeta de desarrollo open-source construida con un microcontrolador modelo Atmega2560 que posee pines de entradas y salidas $(\mathrm{E} / \mathrm{S})$, analógicas y digitales. Esta tarjeta es 
programada en un entorno de desarrollo que implementa el lenguaje Processing/Wiring. Arduino puede utilizarse en el desarrollo de objetos interactivos autónomos o puede comunicarse a un PC a través del puerto serial (conversión con USB) utilizando lenguajes como Flash, Processing, MaxMSP, etc. Las posibilidades de realizar desarrollos basados en Arduino tienen como límite la imaginación.

El Arduino Mega tiene 54 pines de entradas/salidas digitales (14 de las cuales pueden ser utilizadas como salidas PWM), 16 entradas análogas, 4 UARTs (puertos serial por hardware), cristal oscilador de $16 \mathrm{MHz}$, conexión USB, jack de alimentación, conector ICSP y botón de reset. Arduino Mega incorpora todo lo necesario para que el microcontrolador trabaje; simplemente conéctalo a tu PC por medio de un cable USB o con una fuente de alimentación externa (9 hasta 12VDC (Arduino, 2018)

Módulo LM2596 Convertidor de Voltaje DC-DC: este módulo está basado en el Regulador DC-DC Step Down LM2596 que es un circuito integrado capaz de conducir una corriente de hasta 3A. Este circuito te permite tener un voltaje regulado a partir de una fuente de alimentación con un voltaje mayor, por ejemplo, si tienes una fuente de $12 \mathrm{~V}$ puedes regularlos a 5V, 3.3V, 2.2V, etc., para el uso con microcontroladores, drivers para leds, etc.

Transistor Mosfet IRF520: es un dispositivo de estructura metal oxido semiconductor. Un transistor que se controla con voltaje positivo en la compuerta o gate y es usado para amplificar o conmutar señales. Para el proyecto hemos usado la configuración MOSFET Switch permite controlar las cargas.

DFPlayer Mini SKU: DFR0299: el reproductor de MP3 DFPlayer Mini para Arduino es un módulo MP3 con una salida simplificada directamente al altavoz. El módulo se puede usar como un módulo independiente con batería, altavoz y pulsadores adjuntos, o se puede usar en combinación con un Arduino con capacidades $\mathrm{RX} / \mathrm{TX}$.

Modulo Bluetooth HC-05: es un pequeño modulo transmisor/receptor TTL Permite transmitir como recibir datos a través de tecnología bluetooth sin conectar cables a los dispositivos a comunicar. Es un dispositi- vo muy fácil de usar y compacto se controla mediante comandos AT por el puerto serie. Es compatible con Arduino o cualquier microcontrolador con UART.

\section{Software para diseño de circuitos}

EasyEDA: es un conjunto de herramientas EDA basadas en la web que permite a los diseñar, simular, compartir esquemas, simulaciones y placas de circuitos impresos.

Fritzing: es un programa open source para el diseño de esquemas electrónicos. Dispone bibliotecas con la mayoría de componentes incluido los propios Arduinos, placas de conexiones, led, motores, displays, etc. Además, permite diseñar nuestro PCB final y un sinfín de opciones que convierten a este programa en una herramienta muy útil.

\section{Lenguajes de Programación}

Para el desarrollo del software de Control y Seguimiento se ha decidido usar el lenguaje de programación JAVA debido a que es portable, multiplataforma es fácil de programar y posee seguridad en comparación con otros lenguajes.

Java es un lenguaje de programación y una plataforma informática comercializada por primera vez en 1995 por Sun Microsystems. Hay muchas aplicaciones y sitios web que no funcionarán a menos que tenga Java instalado y cada día se crean más. Java es rápido, seguro y fiable. Desde portátiles hasta centros de datos, desde consolas para juegos hasta súper computadoras, desde teléfonos móviles hasta Internet, Java está en todas partes (ORACLE, 2018).

\section{Características y Funcionalidades}

Java le permite jugar, cargar fotografías, chatear en línea, realizar visitas virtuales y utilizar servicios como, por ejemplo, cursos en línea, servicios bancarios en línea y mapas interactivos. Si no dispone de Java, muchas aplicaciones y sitios web no funcionarán (ORACLE, 2018).

\section{Entorno de desarrollo de aplicación}

Para el desarrollo del software se ha seleccionado la plataforma NetBeans ya que estamos familiarizados con la interfaz gráfica que posee lo que para nosotros constituye una alternativa para agilizar el desarrollo de los elementos gráficos y la programación en general. 
NetBeans: es un entorno de desarrollo integrado libre, hecho principalmente para el lenguaje de programación Java. Existe además un número importante de módulos para extenderlo. NetBeans IDE es un producto libre y gratuito sin restricciones de uso.

La plataforma NetBeans permite que las aplicaciones sean desarrolladas a partir de un conjunto de componentes de software llamados módulos. Un módulo es un archivo Java que contiene clases de java escritas para interactuar con las APIs de NetBeans y un archivo especial (manifest file) que lo identifica como módulo.

Las aplicaciones construidas a partir de módulos pueden ser extendidas agregándole nuevos módulos. Debido a que los módulos pueden ser desarrollados independientemente, las aplicaciones basadas en la plataforma NetBeans pueden ser extendidas fácilmente por otros desarrolladores de software. NetBeans IDE soporta el desarrollo de todos los tipos de aplicación Java (J2SE, web, EJB y aplicaciones móviles) (Apache NetBeans, 2016).

Para el desarrollo de la aplicación móvil se ha decidido usar App Inventor.

App Inventor: es un entorno de desarrollo de software creado por Google Labs para la elaboración de aplicaciones destinadas al sistema operativo Android. El usuario puede, de forma visual y a partir de un conjunto de herramientas básicas, ir enlazando una serie de bloques para crear la aplicación. El sistema es gratuito y se puede descargar fácilmente de la web. Las aplicaciones creadas con App Inventor están limitadas por su simplicidad, aunque permiten cubrir un gran número de necesidades básicas en un dispositivo móvil (MIT App Inventor | Explore MIT App Inventor, 2018)

\section{Gestor de Base de Datos}

Para generar la base de datos para el software, se ha empleado la base datos MySQL debido a las características que posee.

MySQL: es un sistema de gestión de bases de datos relacional desarrollado bajo licencia dual: Licencia pública general/Licencia comercial por Oracle Corporation y está considerada como la base datos de código abierto más popular del mundo (MySQL, n.d.)

\section{Características}

- Amplio subconjunto del lenguaje SQL. Algunas extensiones son incluidas igualmente.

- Disponibilidad en gran cantidad de plataformas y sistemas.

- Posibilidad de selección de mecanismos de almacenamiento que ofrecen diferentes velocidades de operación, soporte físico, capacidad, distribución geográfica, transacciones...

- Transacciones y claves foráneas.

- Conectividad segura.

- Replicación.

- Búsqueda e indexación de campos de texto.

\section{Diseño y Construcción de los Módulos}

\section{Escalera Digital Dinámica}

Este módulo como su nombre lo indica es una escalera con funciones interactivas. Posee cinco escalones, cada escalón es un pulsante que cada vez que sea presionado enciende un estímulo visual y auditivo en el siguiente nivel (escalón) con el objetivo de que el niño siga avanzando.

Figura $1 »$ Modelo 3D de Módulo Escalera

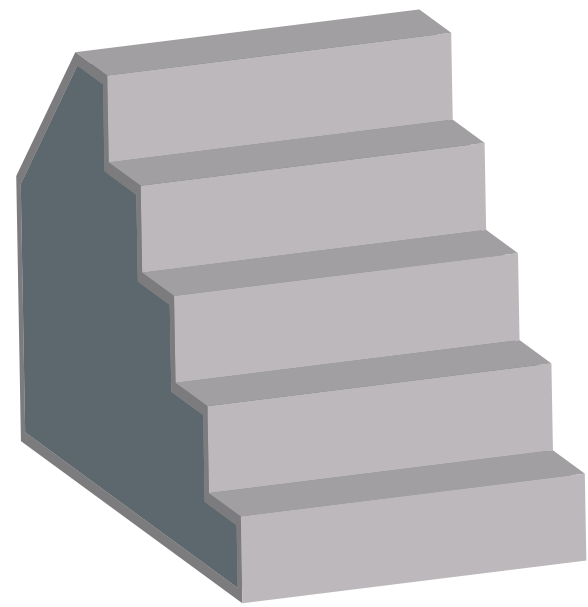

Para tener una idea de las dimensiones reales de la estructura del módulo primero se realizó el diseño 3D, como se puede apreciar en la figura 1. El diseño se hizo con base en dimensiones reales de escaleras destinadas para el uso de terapias con niños. 
Figura 2 » Dimensiones para la Escalera

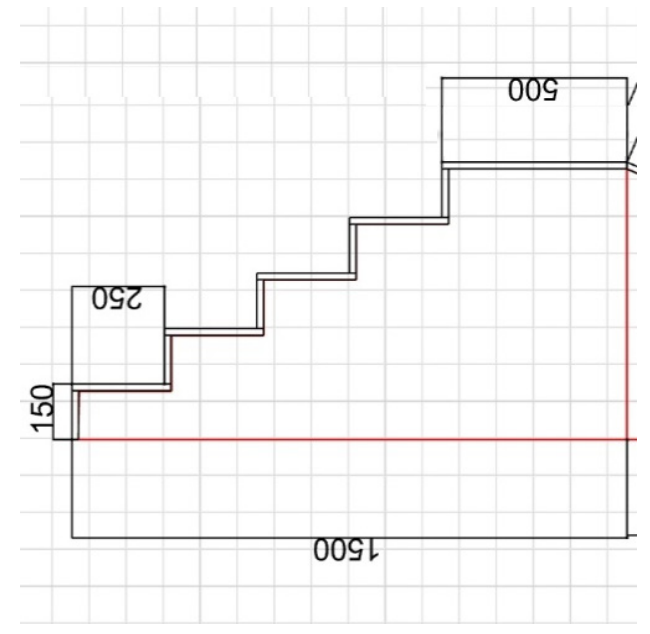

En la construcción de la estructura se utilizó madera, ya que fue la mejor opción para poder hacer una estructura firme y desmontable. Para los pulsantes que se encuentran en la base de cada peldaño se decidió diseñar un pulsante propio y de esta manera abaratar costos porque la otra opción era comprar sensores de presión. El pulsante se realizó a partir del soporte de madera del peldaño, sobre este se agregó dos placas metálicas de las mismas dimensiones, las placas están energizadas, y en medio de estas se encuentra un elemento separador de esta forma cunado se ejerce presión sobre el peldaño estas hacen contacto que cierran el circuito y nos permite desencadenar un evento (sonido y luces). Además, para seguridad de los niños los peldaños y la escalera en general esta acolchonada. Para crear un estímulo visual en la parte frontal de cada peldaño se utilizó acrílico que refleja la luz de una cinta RGB.

\section{Caminadora de Pared}

Este módulo es un panel con pulsantes distribuidos a lo largo de la superficie. Está diseñado con el objetivo de que el niño en sus etapas iniciales de aprender a caminar se apoye en el panel y camine hacia un estímulo visual (Figura luminosa) y cada vez que consiga llegar y tocar la figura se encenderá el siguiente, todo esto acompañado de un sonido de victoria.

Para la construcción de la caminadora de pared se creó un modelo 3D para saber las dimensiones y la forma final que adoptaría. La pared tiene una estructura ondulada debido a que resulta más llamativa para un niño además los relieves generan un nuevo factor de estímulo dentro de la terapia. La estructura del panel es de metal recubierta por planchas de MDF para obtener una superficie firme en la que el niño se pueda apoyar.

Los pulsantes se encuentran colocados a lo largo del frente de la pared a diferentes distancias y alturas permitiendo que el niño tenga más movimiento al momento de ocupar el módulo. Los diseños de los pulsantes de la caminadora de pared son similares a los de la escalera con la diferencia de que estos se encienden. Están construidos con dos placas metálicas una base de madera y en la parte frontal un acrílico con el dibujo de una mano. Al igual las placas metálicas están energizadas y entre ellas tienen un elemento separador, cuando se ejerce una presión cierran el circuito y permiten encender la cinta RGB que se encuentra en la parte frontal sobre el acrílico.

El módulo de pared busca ser entretenido para el niño ayudándole a desarrollar su motricidad gruesa.

\section{Figura 3 » Caminadora de pared Funcionamiento}

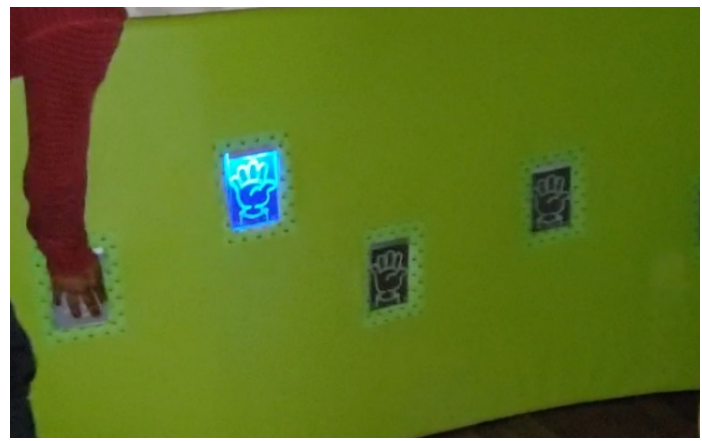

\section{Diseño del circuito electrónico}

El circuito electrónico está diseñado para el control de los eventos de los dos módulos respectivamente. Para la elaboración del circuito se diseñó un circuito esquemático en el software de diseño Fritzing y para el diseño del PCB se utilizó EasyEDA. Además, se realizó una serie de pruebas en el Protoboard con el propósito de asegurar el circuito funciones conjuntamente con los módulos. Luego de realizar las pruebas necesarias se inició la construcción de la placa, la cual es el centro de procesamiento de los módulos debido a que controlara los diferentes eventos como iluminación y sonidos. 
El microcontrolador está programado de tal forma que cuando se active una entrada, este activará la salida correspondiente y reproducirá un sonido. También está programado para controlar la secuencia y variación de las luces de cada módulo. A continuación, se presenta un diagrama de flujo de cómo está configurado el microcontrolador en relación con los pulsantes.

\section{Figura 4 » Diagrama de Flujo (Evento de los} Pulsantes)

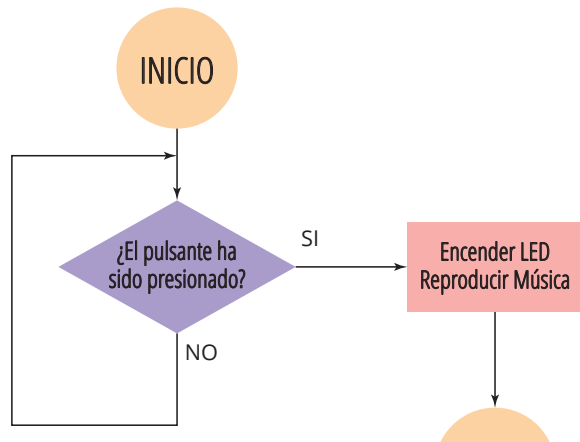

FIN

\section{Diseño del Software de Control y Seguimiento}

Después de analizar las necesidades de los terapeutas se decidió realizar un software para el control y seguimiento de las terapias realizadas con el propósito de medir las interacciones de los niños con los módulos y registrarlas en una base de datos que permita su posterior análisis y seguimiento. Los datos que se almacenan permitirán obtener información para conocer el progreso de cada niño a lo largo de las terapias.

Para el diseño y desarrollo del software se realizó el análisis respectivo. El software servirá como herramienta para que el profesional pueda gestionar la sesión de terapia para cada niño. De igual forma es importante llevar un registro de las terapias para conocer el progreso del niño en cada terapia por lo que el programa cuenta con una base de datos en donde se guardara información obtenida en cada terapia. El análisis se realizó a partir del diagrama de casos de uso del software de control y seguimiento.

Figura 5» Diagrama Casos de Uso del software de control y seguimiento

Sistema de Control y Seguimiento

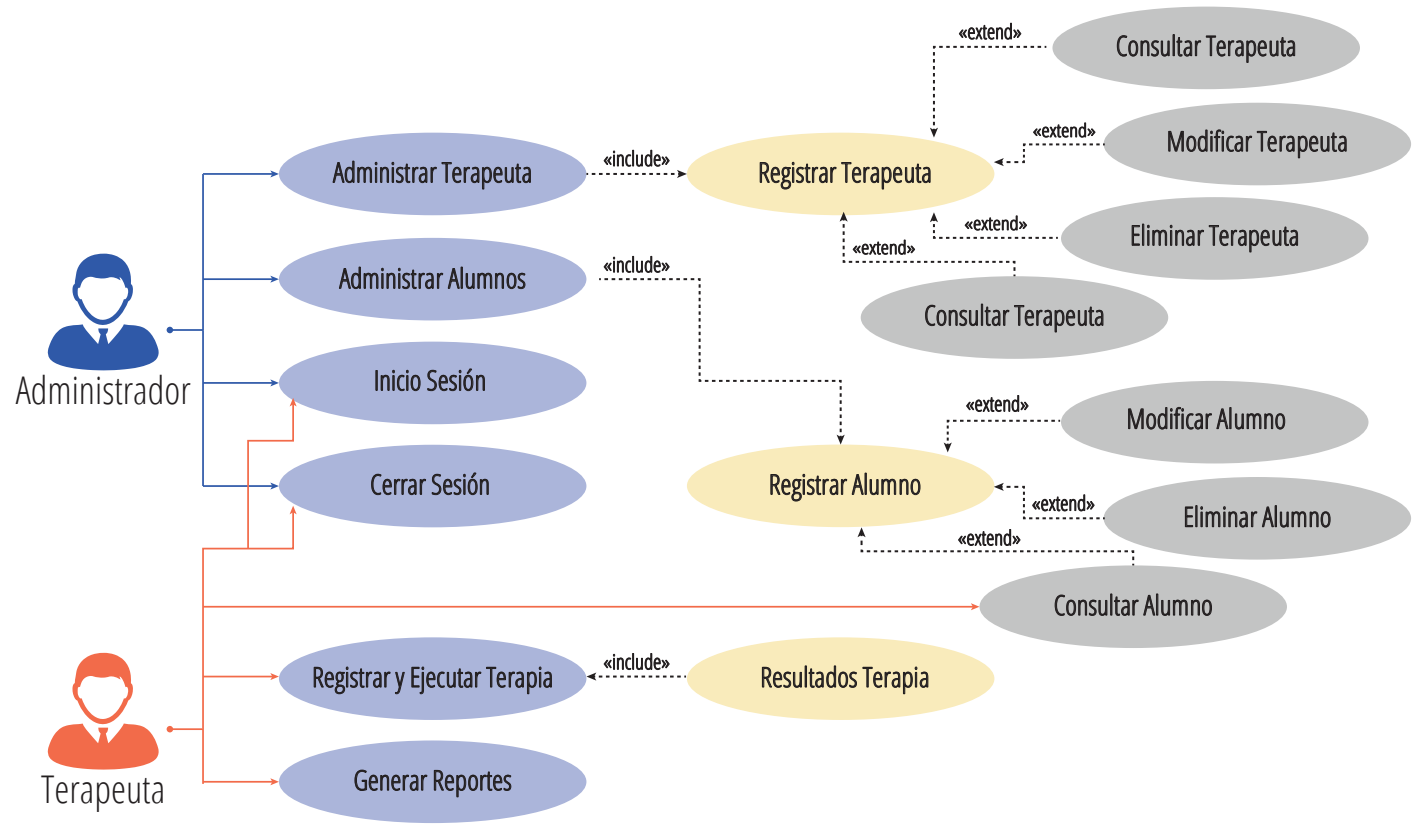

Para el desarrollo del software de Control y es fácil de programar y posee seguridad en comparaSeguimiento se ha decidido usar el lenguaje de progración con otros lenguajes. 


\section{Interfaz Gráfica del Software}

\section{Figura 6» Interfaz Gráfica}
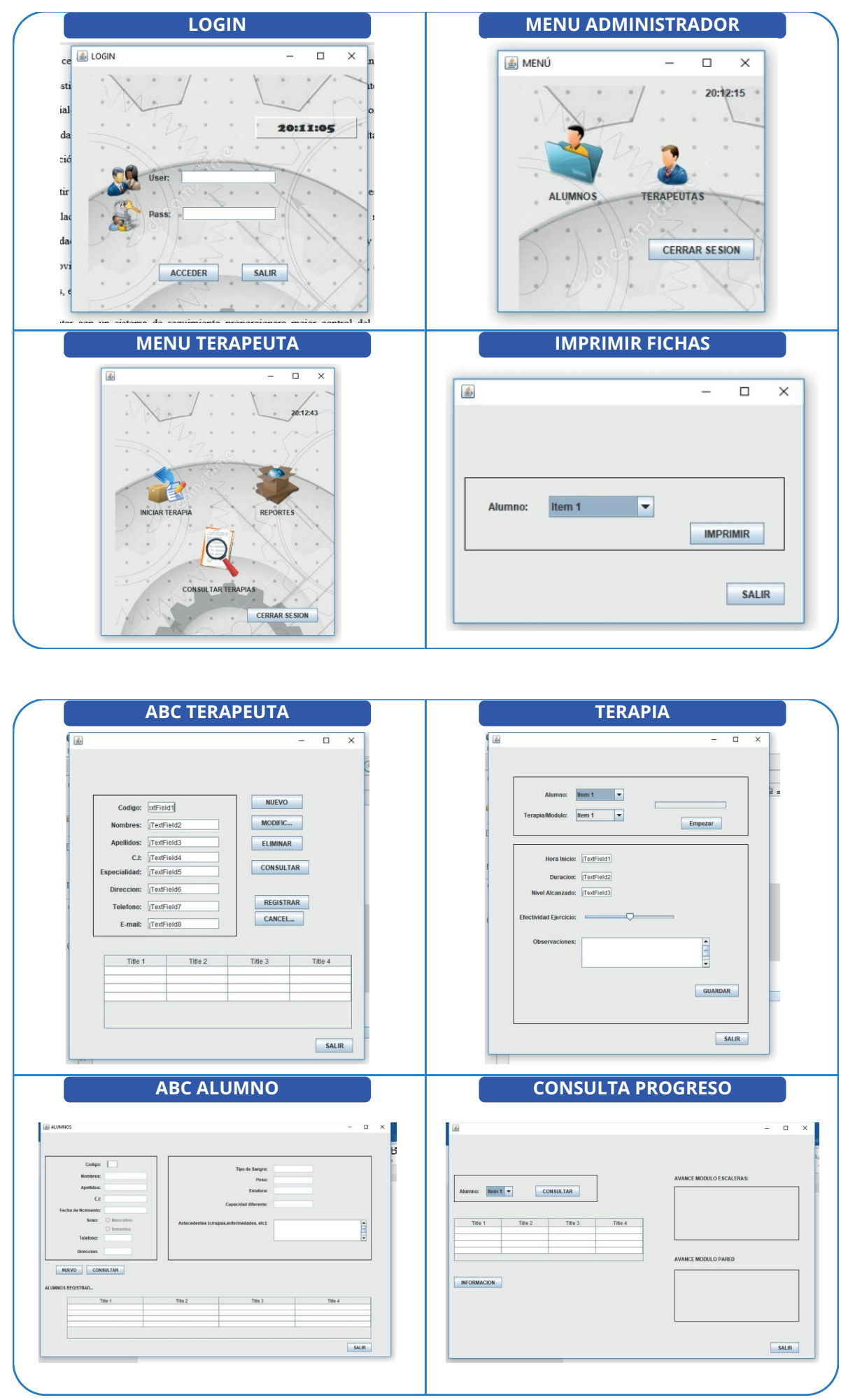

Espacio multisensorial para la estimulación de niños entre dos y cinco años con déficit sensorial 


\section{Diseño de la Aplicación Móvil}

Para diseñar la aplicación de Android ocupamos el entorno de desarrollo APP Inventor debido a la compatibilidad en la comunicación con el módulo Bluetooth
HC-05 y el dispositivo móvil. Antes de iniciar con la programación en Android se hizo el análisis para la creación de esta Aplicación. Esta aplicación está destinada al control de la variación de luces y sonidos.

Figura 6» Diagrama casos de uso de la App Móvil

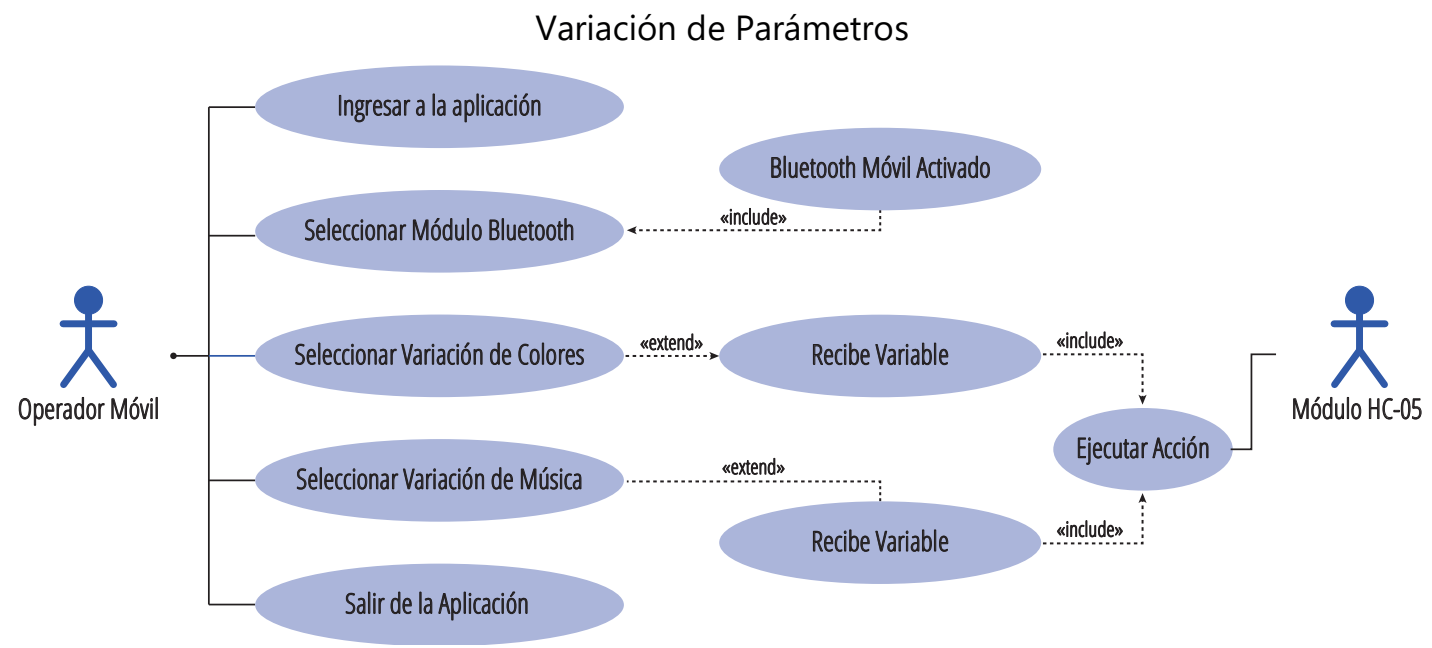

Figura 8 » Diagrama de Flujo de la App Móvil

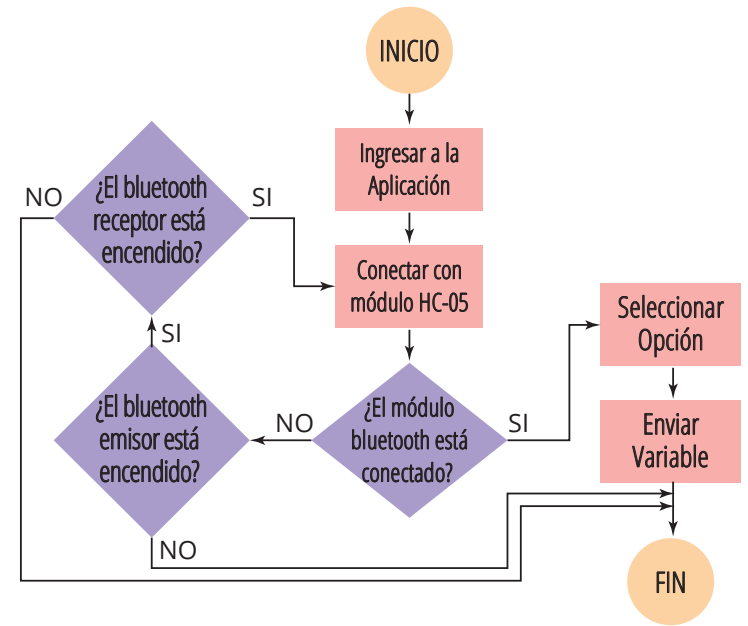

Una vez que el móvil mediante la aplicación se conecta al Módulo hc-05 podemos enviar las variables programadas 'a', 'b','c' para que el Modulo las reciba y el Arduino ejecute una sentencia siempre que se cumpla una condición.

Para el diseño de la aplicación optamos por un sistema más minimalista enfocado a una interfaz de botones dedicada controlar las variaciones de los módulos.
Figura 9» Programación de la App Móvil

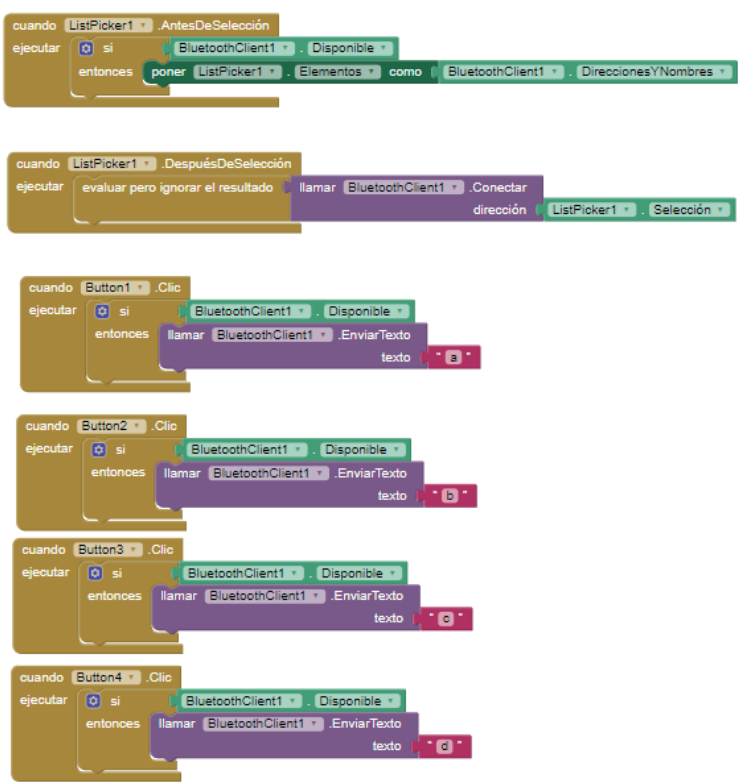

\section{CONCLUSIONES}

Tras la culminación del proyecto "DISEÑO Y CONSTRUCCIÓN DE DOS MÓDULOS DIGITALES PARA LA ESTIMULACIÓN SENSORIAL Y MOTRIZ (GATEAR, CAMINAR) DE NIÑOS DE 2 A 5 AÑOS" se ha llegado a las siguientes conclusiones: 
- Los módulos están destinados con la finalidad de convertirse en una herramienta educativa, para niños en etapas iniciales de vida ( 2 a 6 años) debido a que en esa edad ya empiezan a caminar.

- Este trabajo está basado en fundamentos teóricos de varios autores para su elaboración. Para la construcción de los módulos se tomaron en cuenta las principales teorías sobre la estimulación temprana, el desarrollo psicomotor, la estimulación multisensorial y la plasticidad neuronal.

- Dado que los módulos van a ser usados por niños fue necesario considerar aspectos como el acolchonado, elementos de protección para las conexiones y demás materiales durante la construcción con el fin de que los módulos sean seguros para los niños. No obstante, cualquier persona que no esté familiarizada con el software de control y seguimiento puede utilizarlo ya que posee una interfaz fácil de comprender e intuitiva.

\section{RECOMENDACIONES}

- La estimulación temprana es muy importante para el desarrollo del niño, pero sería optimo experimentar en nuevas áreas de educación diseñando nuevos dispositivos de apoyo.

- Se considera factible ampliar el proyecto con nuevos módulos que contribuyan en nuevas áreas de terapia.

- Implementar estos módulos en una institución en la que se puedan usar en las terapias para obtener resultados de su funcionalidad.

- A futuro se espera por retroalimentación implementar más opciones en el software de acuerdo a los requerimientos una vez los módulos entren en funcionamiento.

\section{REFERENCIAS BIBLIOGRÁFICAS}

Aguilar Cordero, M. J., Vieite Ravelo, M., Padilla López, C. A., Mur Villar, N., Rizo Baeza, M., \& Gómez García, C. I. (2012). La estimulación prenatal; resultados relevantes en el periparto. Nutricion Hospitalaria, 27(6), 2102-2108. https://doi.org/10.3305/ nh.2012.27.6.6019
Apache NetBeans. (2016). Welcome to Apache NetBeans. https://netbeans.apache.org/

Arduino. (2018). What is Arduino? | Arduino. https:// www.arduino.cc/en/Guide/Introduction

ASIN, S. (1989). fundamentos biológicos educación bases intervención de sánchez asín Iberlibro. https://www.iberlibro.com/servlet/ SearchResults?an=s\%E1nchez as\%EDn\&tn=fundamentos biol\%F3gicos educaci\%F3n bases intervenci\%F3n\&cm_sp=mbc-_-ats-_-all

BERRUEZO ADELANTADO, P. P. (2008). El contenido de la Psicomotricidad. Reflexiones para la delimitación de su ámbito teórico y práctico. Revista Interuniversitaria de Formación Del Profesorado, Vol. 22, Núm. 2, Agosto, 2008, Pp.19-34 Universidad de Zaragoza Zaragoza, España. https://www.redalyc.org/pdf/274/27414780003.pdf

Coronel, L. M. P. (2014). UNIVERSIDAD CENTRAL DEL ECUADOR FACULTAD DE CIENCIAS PSICOLÓGICAS.

Gardner, H. (2016). Amazon.com: Frames of Mind: The Theory of Multiple Intelligences (9780465024339): Gardner, Howard E: Books. https://www.amazon. com/-/es/Howard-Gardner/dp/0465024335

GLENN DOMAN, DOUGLAS DOMAN, B. H. (2012). Bebé en forma, bebé inteligente. Potenciar la movilidad temprana en los bebés para favorecer el desarrollo cerebral y físico - Editorial Edaf S.L.U. https://www.edaf.net/libro/bebe-en-forma-bebe-inteligente_88499/

Gómez, M. del C. G. (2012). Aulas multisensoriales en educación especial: estimulación e integración sensorial en los espacios snoezelen (Libro, 2012) [WorldCat.org]. https://www.worldcat.org/title/ aulas-multisensoriales-en-educacion-especial-estimulacion-e-integracion-sensorial-en-los-espacios-snoezelen/oclc/851181099

Hernández, J. R. (2019). Actividades para estimular a los niños, de acuerdo con la edad. https://www. eltiempo.com/abc-del-bebe/bebe/0-6-meses/actividades-para-estimular-a-los-ninos-de-acuerdo-con-la-edad-13344

Jesús, A., \& Sánchez, P. (2005). "ESQUEMA CORPORAL Y LATERALIDAD.” ESQUEMA CORPORAL. 


\section{CONVERGENCE TECH}

Katerinesisalima, B., María, P., Vanegas Vintimilla, F., De Psicología, F., Cesibel, M., \& Pineda, O. (2013). Importancia del desarrollo sensorial en el aprendizaje del niño.

Keller, H., Yovsi, R. D., \& Voelker, S. (2002). THE ROLE OF MOTOR STIMULATION IN PARENTAL ETHNOTHEORIES The Case of Cameroonian Nso and German Women.

Lalama Moreira Alvarez, C. (2016). Efectos de la estimulación multisensorial dentro de una sala oscura en el tratamiento del equilibrio en niños de 5 a 7 años con discapacidad intelectual. Quito: Universidad de las Américas, 2016. http://dspace.udla.edu.ec/ handle/33000/6270

Miranda, C., \& Yracema QUIÑA SEDANO, J. (2017). "AÑO DEL BUEN SERVICIO AL CIUDADANO" UNIVERSIDAD NACIONAL DE HUANCAVELICA (Creada por la Ley $\mathrm{N}^{\circ}$ 25265) FACULTAD DE EDUCACIÓN PROGRAMA DE SEGUNDA ESPECIALIDAD DE EDUCACIÓN INICIAL 1.1.1.1.1.1.1 INFORMEDEL TRABAJO ACADÉMICO PRESENTADO POR: ESTIMULACIÓN SEN.

MIT App Inventor | Explore MIT App Inventor. (2018). https://appinventor.mit.edu/

MySQL. (n.d.). from https://www.mysql.com/

ORACLE. (2018). ¿Qué es Java? https://www.java.com/ es/about/whatis_java.jsp

Orlando Schrager, L. (1986). Las apraxias en el infante y en el niño. "Revista de Logopedia, Foniatria y Audiologia," 6(3), 129-143. https://doi.org/10.1016/ S0214-4603(86)75377-1

Santos, S. N. L. (2012). UNIVERSIDAD TÉCNICA DE AMBATO FACULTAD DE CIENCIAS HUMANAS Y DE LA EDUCACIÓN CARRERA DE: EDUCACIÓN PARVULARIA "LA ESTIMULACIÓN TEMPRANA Y SU INCIDENCIA EN EL.

Vega, C. V. C. (2016). La estimulación temprana en el desarrollo infantil de los niños y niñas del primer ciclo de educación inicial | Semantic Scholar. https://www.semanticscholar.org/paper/Laestimulación-temprana-en-el-desarrollo-infantilVega/bab88cfd71851e7482782819df90cc728cfd9598 